\title{
Evaluación del riesgo cardiovascular sin medición de la colesterolemia
}

\author{
Cardiovascular risk evaluation without colesterol determination
}

Gaziano T y col. Lancet 2008; 371:923-31.

\section{Objetivo}

Comparar en una cohorte real, una regla de predicción clínica para enfermedad cardiovascular que no incluye testeo de laboratorio con otra que sí lo incluye.

Diseño

Diseño y validación de una regla de predicción clínica.

\section{Lugar}

Cohorte de estudio de seguimiento del National Health and Nutrition Examination Survey (NHANES) estadounidense, denominado NHEFS.

\section{Pacientes}

Se usó un muestreo multietápico estratificado* para seleccionar una muestra representativa de individuos estadounidenses que tuvieran entre 25 y 74 años cuando fueron examinados por primera vez, entre 1971 y 1975 . Fueron incluidos 6186 participantes con información completa, que no reportaron antecedentes de enfermedad cardiovascular (infarto de miocardio, insuficiencia cardiaca, accidente cerebrovascular o angina de pecho)

\section{Evaluación de los factores pronósticos}

El estudio comparó qué tan bien cada método podía predecir la ocurrencia de eventos cardiovasculares fatales y no fatales. Para el modelo basado en laboratorio que requería análisis de sangre, se utilizaron los factores de riesgo clásicos para evaluar la probabilidad de enfermedad cardiovascular como edad, presión sistólica, estatus de fumador, colesterol total, estatus de diabético reportado, colesterol total y tratamiento actual para dislipidemia. En el modelo no basado en laboratorio se sustituyó el colesterol por índice de masa corporal (IMC). Ambos modelos fueron comparados a través de una regresión de Cox.

\section{Medición de resultado principal}

La discriminación predictiva se evaluó en una serie de características como sensibilidad, especificidad, valores predictivos positivos y negativos y curvas ROC*. Esta curva mide la discriminación de un modelo de predicción y es la gráfica de la tasa de verdaderos positivos versus la de falsos positivos. El estadístico $\mathrm{c}$ representa un estimador de la probabilidad de que el modelo asigne un riesgo mayor a aquellos individuos que presentan eventos, en relación a aquellos que no. Estas características fueron evaluadas a cuatro niveles diferentes de riesgo a cinco años de eventos $(5 \%, 10 \%$, $20 \%$ y $30 \%$ ).

\section{Resultados principales}

Existieron 1529 nuevos eventos cardiovasculares y 578 muertes por enfermedad cardiovascular en 21 años de seguimiento. En mujeres, el modelo basado en laboratorio fue útil para predecir eventos, con un estadístico $c$ de 0,829 mientras que para el método no basado en laboratorio fue de 0,831 . En hombres los resultados fueron similares $(0,784$ y 0,783 respectivamente). La tabla 1 describe las características operativas de la herramienta desarrollada en este estudio, cuyas tablas para calcular los puntajes acercamos al lector en la actualización sobre riesgo cardiovascular que publicamos a continuación en este mismo ejemplar de EVIDENCIA.

Tabla 1: discriminación del puntaje sin colesterolemia a diferentes umbrales de riesgo cardiovascular a cinco años

\begin{tabular}{|c|c|c|c|c|c|c|}
\hline \multicolumn{3}{|c|}{ Garacterísticas operativas } & $5 \%$ & $10 \%$ & $20 \%$ & $30 \%$ \\
\hline \multirow[t]{4}{*}{ Mujeres } & \multicolumn{2}{|c|}{ Sensibilidad } & $76,4(73,2$ a 79,5$)$ & $53,5(49,8$ a 57,2$)$ & $17,6(15,0$ a 20,8$)$ & $5,1(3,6$ a 7,0$)$ \\
\hline & \multicolumn{2}{|l|}{ Especificidad } & $73,5(71,8$ a 75,2$)$ & $88,4(87,1$ a 89,6$)$ & $97,7$ (97,1 a 98,3$)$ & $99,5(99,2$ a 99,7$)$ \\
\hline & \multirow[t]{2}{*}{ Valor predictivo } & Positivo & $44,2(41,4$ a 47,0$)$ & $55,9(52,2$ a 59,6$)$ & $68,1$ (60,9 a 74,7$)$ & $74,0(59,7$ a 85,4$)$ \\
\hline & & Negativo & $91,9(90,7$ a 93,0$)$ & $87,4(86,1$ a 88,6$)$ & $81,2(79,8$ a 82,6$)$ & $80,4(79,0$ a 1,7$)$ \\
\hline \multirow[t]{4}{*}{ Hombres } & \multicolumn{2}{|l|}{ Sensibilidad } & $84,8(82,4$ a 87,1$)$ & $60,2(56,9$ a 63,5$)$ & $24,8(21,9$ a 27,8$)$ & $8,8(7,0$ a 10,9$)$ \\
\hline & \multicolumn{2}{|l|}{ Especificidad } & $56,7(54,5$ a 59,0$)$ & $78,3(76,4$ a 80,1$)$ & $93,7$ (92,5 a 94,7$)$ & $98,6(98,0$ a 99,1$)$ \\
\hline & \multirow[t]{2}{*}{ Valor predictivo } & Positivo & $46,5(44,0$ a 49,0$)$ & $55,2(52,0$ a 58,4$)$ & $63,5(58,2$ a 68,7$)$ & $74,4(64,5$ a 82,1$)$ \\
\hline & & Negativo & $89,3(87,5$ a 91,0$)$ & $81,6$ (79,8 a 83,3$)$ & $73,7(72,0$ a 75,5$)$ & $70,9(69,2$ а 72,6$)$ \\
\hline
\end{tabular}

\section{Conclusiones}

El método que utiliza factores de riesgo no basados en laboratorio predijo eventos cardiovasculares con tanta precisión como el que incluye valores de laboratorio.

\section{Palabras clave: riesgo cardiovascular, pronóstico.}

Key words: cardiovascular risk, prognosis.

Fuente de financiamiento: Fogarty International Center grant from the National Institutes of Health.

\section{Comentario}

Este estudio implica un movimiento positivo hacia un proceso de rastreo simplificado del riesgo cardiovascular global, sobre todo en escenarios con escasos recursos, como es el caso en varios países latinoamericanos. La Organización Mundial de la Salud (OMS) recientemente ha lanzado guías para la prevención de la enfermedad cardiovascular que incluyeron conjuntos de tablas de evaluación para diferentes regiones mundiales, con y sin métodos de laboratorio'. Sin embargo estas estimaciones no estuvieron basadas en eventos registrados prospectivamente, y aún no han sido extensamente validadas. El presente trabajo, en cambio, com- para modelos en individuos seguidos a largo plazo en una cohorte real, como la del estudio NHANES, en más de 6000 personas y demuestra que ambos métodos son igualmente exactos en su capacidad predictiva. En Argentina, no existen al momento cohortes con representatividad nacional que registren la ocurrencia de eventos cardiovasculares. Sin embargo, y si bien los riesgos basales de otros países no son necesariamente trasladables, esta aproximación en su concepción podría simplificar la evaluación del riesgo en nuestras poblaciones a partir de datos provenientes de encuestas de representatividad nacional sin datos bioquímicos.

Ariel Bardach [ Instituto de Efectividad Clínica y Sanitaria. abardach@iecs.org.ar ]

Bardach A. Evaluación del riesgo cardiovascular sin medición de la colesterolemia. Evid. actual. práct. ambul; 11(3): 77, May-Jun 2008. Comentado de: Gaziano T. Laboratory-based versus non-laboratory-based method for assessment of cardiovascular disease risk: the NHANES I Followup Study cohort. Lancet 2008; 371:923-31.

\section{Referencias}

1. World Health Organization. Prevención de enfermedades cardiovasculares. Guía de Bolsillo para la Estimación y el Manejo del Riesgo Cardiovascular: Americas. Ginebra,2007 ISBN-13 9789243547282 ISBN-10 924354728 En prensa: http://www. who.int/bookorders/espagnol/detart3.jsp?sesslan=3\&codlan=3\&codcol=15\&codcch=4713\# 